\title{
El cuidado de niños sin cuidado parental en contextos escolares y de protección ${ }^{8}$
}

\author{
María Rosa Estupiñán Aponte \\ Doctora en Ciencias Sociales, Niñez y Juventud \\ Universidad Pedagógica y Tecnológica \\ de Colombia, Tunja. Colombia \\ Correo electrónico: mrestupinana@unal. \\ edu.co, maria.estupinan@uptc.edu.co
}

\section{Fred Gustavo Manrique Abril}

Doctor en Salud Pública y en Medicina Clínica Universidad Pedagógica y Tecnológica de Colombia, Tunja. Colombia Universidad Nacional de Colombia, Sede Bogotá, Colombia

Correo electrónico: fgmanriquea@unal.edu.co
Recibido: $12 / 09 / 2018$

Evaluado: $15 / 11 / 2018$

Aceptado: 27/11/2018

"...lo que el gobierno quiere es que cuidemos los niños, no es tanto que les enseñemos... y los papitos también están en ese plan..."

\section{Resumen}

En el mundo millones de niños crecen sin el cuidado de sus padres planteando grandes retos a las instituciones escolares y de protección, en su función socializadora. A través de observación participante, entrevistas y relatos de vida, el estudio buscó abordar los significados del cuidado de niños sin cuidado parental, otorgados por funcionarios del Estado, maestros y personal de servicios, a las condiciones de vida, relaciones que establecen y formas como negocian su situación. Las perspectivas que acompañan las acciones de cuidado por parte de la población investigada, entrecruzan categorías relacionadas con la supervivencia física y el acompañamiento emocional que les suponen establecer acuerdos, reconocer las propias limitaciones y actuar en consecuencia, dando lugar a tres formas de vivir el cuidado: como distribución de recursos, como carga laboral y como vínculo afectivo. Así, el cuidado involucra acciones, relaciones y marcos normativos, económicos y sociales que desbordan el rol asignado.

\section{Palabras clave}

Abandono infantil, cuidado del niño, ética del cuidado, educación infantil.

8 Para citar este artículo: Estupiñán, M.R., \& Manrique-Abril, F.G. (2019). El cuidado de niños sin cuidado parental en contextos escolares y de protección. Informes Psicológicos, 19(2), pp. 109-124http://dx.doi.org/10.18566/infpsic. v19n2a08 


\title{
The care of children without parental care in school and protection contexts
}

\begin{abstract}
Millions of children grow up without the care of their parents in the world today, which poses great challenges to school and protection institutions, in their socializing role. Through participant observation, interviews and life stories, the study sought to address the meanings of childcare without parental care that State officials, teachers and service personnel give to the living conditions, the relationships they establish, and the ways in which they negotiate their situation. The perspectives that accompany the actions of care on the part of the investigated population intersect categories related to physical survival and emotional accompaniment that involve establishing agreements, recognizing one's limitations and acting accordingly, giving rise to three ways of living care: as a distribution of resources, as a workload and as an affective bond. Thus, care involves actions, relationships and normative, economic and social frameworks that go beyond the assigned role.
\end{abstract}

\section{0 cuidado das crianças sem cuidado parental em contextos escolares e de proteção}

\section{Resumo}

No mundo milhões de crianças crescem sem os cuidados de seus pais dando origem a grandes retos para as instituições escolares e aos serviços de proteção em sua função socializadora. Por meio de observação participante, entrevistas e relatos de vida, este estudo procurou abordar os significados do cuidado de crianças sem cuidado parental, fornecido por funcionários do estado, mestres e pessoal de serviços, às condições de vida, às relações que estabelecem e formas nas quais negociam com sua citação. As perspetivas que acompanham as ações de cuidado por parte da população pesquisada, entrelaçam categorias relacionadas com a sobrevivência física e 0 acompanhamento emocional que supõe-Ihes estabelecer acordos, reconhecer as próprias limitações e atuar em consequência, dando origem a três formas de viver o cuidado: como distribuição de recursos, como carga de trabalho e como vinculo afetivo. Assim, o cuidado envolve ações, relações e quadros normativos, econômicos e sociais que vão além do papel atribuído. 


\section{ntroducción}

A pesar de las implicaciones que ha tenido la Convención de Derechos del Niño (CDN) como instrumento político y jurídico que regula el campo de la infancia y la adolescencia, el reconocer a los niños como sujetos sociales de derechos, también ha dado lugar a cuestionamientos sobre las explicaciones de su vida, su comportamiento, la forma como son vistos en la sociedad, el rol que pueden desempeñar como seres humanos activos en ella y el papel que deben cumplir los agentes designados para su cuidado.

La noción de cuidado involucra acciones y relaciones orientadas a cubrir las necesidades físicas y emocionales de los niños, así como los requerimientos legales, económicos y sociales que reglamentan su implementación (Daly \& Lewis, 2000), ya que no pueden ser entendidos aislados de los contextos en que crecen, como una abstracción del mundo que les rodea o de sus circunstancias especiales. El ingrediente emocional del cuidado se exacerba en este proceso cuando los niños no cuentan con el cuidado de sus padres ni tienen las filiaciones necesarias para desarrollar sus vínculos sociales a través de lazos de pertenencia, interdependencia e identificación que les permitan vivir una experiencia de seguridad y protección, los eduquen y preparen para la vida, así como para comprender y compartir lo que significa el vínculo social.
En Europa Central y del Este, aproximadamente 1,3 millones de niños viven en instituciones públicas y casi la mitad en centros de cuidados residenciales o en internados (Unicef, 2006). En tanto que en América Latina cientos de miles de niños carecen de cuidados parentales y no tienen garantizados los elementos necesarios para su crecimiento tales como formar parte de un grupo que los acepte, les exprese cariño, los valore y respete sus derechos (Pinheiro, 2012).

Para Colombia, la Red de Acogimiento Familiar RELAF (2011) señala que muchos niños transcurren su niñez y cumplen la mayoría de edad en instituciones de protección impactando la construcción de lazos afectivos y de capacidades para la vida que les permitan su inclusión social; también documenta que la mayor concentración de niños sin cuidado parental (NSCP) se da en escolares de 10 a 14 años, el 11.2\% de la población infantil total, seguido por los niños entre 5 y 9 años, que representan el 8.2\%.

Esta situación presenta al Estado colombiano el reto de establecer las formas más apropiadas de ofrecer a NSCP los cuidados que necesitan y la educación que les permita "devenir adulto", así como construir su identidad como personas que permanecen en estrecha relación con sus entornos socioculturales. Para ello, ha descentrado del enfoque familiar las funciones de cuidado, dando lugar al posicionamiento de las instituciones educativas y de protección de NSCP, como contextos necesarios para garantizar su sobrevivencia e integración sociocultural, constituyéndose en alternativa para su cuidado. 
No obstante, la complejidad de sus funciones ha provocado la emergencia de disputas y señalamientos, pero especialmente el descargue de responsabilidades que van tejiendo un limbo, una intrincada red de caminos y posibilidades por donde deambulan NSCP buscando su lugar, y donde maestros, personal de servicios y funcionarios de la Policía de Infancia y el Instituto Colombiano de Bienestar Familiar ICBF, van constituyéndose en apoyos necesarios para su cuidado, con las implicaciones de interés, atención, vínculos afectivos y emocionales que le son inherentes.

Un punto de partida para comprender las dinámicas que caracterizan el acompañamiento de NSCP podría estar relacionado con el reconocimiento de las diferentes perspectivas de cuidado que se entrelazan en las relaciones en la Escuela o los Centros de Protección como espacios de crecimiento.

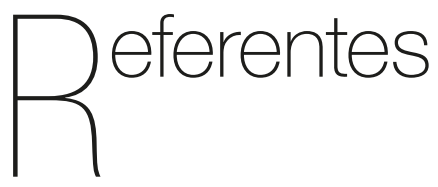

Aunque son múltiples las causas por las que millones de niños no cuentan con el cuidado de sus padres, el abandono y el maltrato intrafamiliar se mantiene pasmosamente desde épocas primordiales de la historia del hombre, a pesar de las reiteradas invitaciones, como es el caso de la "Convención sobre los Derechos del Niño", a reconocer la importancia de la convivencia familiar y comunitaria para el crecimiento y el bienestar de todos sus miembros, en particular el de los niños (Rodríguez \& Franco, 2013).
Es por ello que, cuando la propia familia del niño no puede, ni siquiera con un apoyo apropiado, proveer su cuidado, o cuando lo abandona o renuncia a su guarda, por disposición de los estados, resultante o no de medidas judiciales o administrativas, los centros de protección ofrecen o tramitan acogimiento alternativo a los NSCP en un entorno residencial que circunscribe a los centros de acogida. Como señalan Rodríguez y Franco (2013), oficializar una institución, de parte del Estado, supone el establecimiento de normas de funcionamiento, jerarquía de puestos y funciones, así como atender a la promoción y salvaguardia de los derechos de los NSCP, incluyendo el acceso a la educación (Naciones Unidas, 2010). Así, se implementan acciones de cuidado, generalmente enfocadas a garantizar la sobrevivencia y protección.

No obstante, la noción de cuidado ha presentado diversos matices que es necesario retomar para la comprensión de las perspectivas con que docentes y funcionarios acompañan el crecimiento de los NSCP. Al respecto, Heidegger (2000) plantea el cuidado como actitud que caracteriza la condición de humanidad, mientras que Boff (1999; 2002) propone ampliar este punto de vista en el plano ecológico, destacando el papel del ser humano como parte del mundo de la vida. Así mismo, Gilligan (1985), en perspectiva de género, asume el cuidado como una condición ética (care) que caracteriza la convivencia social, coincidiendo con Tronto (2010) en articular el cuidado a las acciones humanas encaminadas a preservar el mundo garantizando su mantenimiento, reparación y perpetuación. 
Esta perspectiva es compartida también por Terrón (2010) quien, desde la ética del cuidado, introduce el término responsividad como concepto moral que involucra el altruismo como dispositivo necesario en la atención a las condiciones de vida de los "otros", que no acaba en la intención de ayuda, sino que los aborda más allá de las emociones que se viven (Blum, 1994). Estos encuentros humanos en ocasiones son recíprocos permitiéndoles establecer y mantener las condiciones que faciliten el desarrollo de las capacidades de cada uno/a (Noddings, 2003).

Las implicaciones del cuidado en contextos educativos y de protección debería entonces involucrar la atención a las necesidades de las NSCP en cuanto seres vulnerables e interdependientes que construyen y proyectan sus vidas. Relatos de los NSCP ubican en algunas de las personas encargadas de su protección y/o educación, conductas excepcionales caracterizadas por virtudes como la simpatía, la compasión, la amabilidad, la generosidad, la preocupación (Comins, 2008), el apoyo, el afecto y el reconocimiento de sus necesidades.

De esta forma, el cuidado implicaría actividades orientadas al fortalecimiento de valores que favorecen el bienestar humano y que pueden ser aprehendidas de múltiples maneras (Noddings, 2003), evidenciando la necesaria relevancia de los efectos sensibles que posibilitan los vínculos afectivos y relacionales del cuidado (Cerri \& Alamillo, 2012), así como la importancia de tener en cuenta las necesidades sentidas que demandan de los cuidadores relaciones de auténtica empatía y compromiso (Gilligan,1985), más allá de las iniciativas individuales, hacia el ámbito político y, de ahí, al contexto global de la vida social (Robinson, 1999).

Las diferencias o similitudes entre el ideal del cuidado y la práctica están permeadas por las diferentes perspectivas con que los actores abordan las relaciones con NSCP, en los contextos donde tienen lugar, ya que, como afirma Ortega y Gasset (1986), el punto de vista de cada individuo es esencial e insustituible, $y$, aunque complementario, no es idéntico al de las demás personas, es decir, lo que para unos resulta importante, para otros puede carecer de sentido. De esta forma, todas las perspectivas son válidas y dan cuenta de la realidad, formando parte de las diferencias individuales, las peculiaridades de cada persona y del momento histórico. Fundamento que acompañó el estudio al abordar los significados otorgados por funcionarios, docentes y personal de servicios, al cuidado de NSCP.

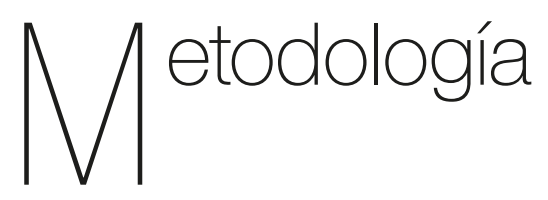

La investigación cualitativa multisituada como método para el estudio de las condiciones sociales (Marcus, 2001), posibilitó el abordaje de las experiencias de acompañamiento al crecimiento de NSCP y familiarizarse con el significado de la interacción, conexiones y relaciones presentes en los ámbitos y vínculos que los participantes establecen. Se desarrolló en las ciudades de Duitama, Sogamoso y el corregimiento de Nazareth en Nobsa, en el departamento de 
Boyacá (Colombia), participando tres policías de infancia, seis funcionarios del Instituto Colombiano de Bienestar Familiar (ICBF) responsables de servicios y atención en los centros zonales, diecisiete docentes de educación básica y ocho empleados del personal de servicios, tales como celadores, jardineros, cocineras y auxiliares en centros de protección, como la Fundación Baudilio Acero (FBA) y el Hogar del Divino Niño (HDN), en Sogamoso.

El estudio se desarrolló en tres fases: fase exploratoria de aproximación al territorio e identificación de informantes clave, se entrevistó a 3 funcionarios de la Policía de Infancia y se visitaron los Centros zonales en las ciudades de Tunja, Duitama y Sogamoso, en el departamento de Boyacá. Allí se entrevistaron los coordinadores regionales y se obtuvo el permiso para la visita a los centros de protección en la ciudad de Sogamoso. La información obtenida también permitió la identificación de dos instituciones educativas, una de básica primaria y otra de básica secundaria, que cuentan con un gran número de población de NSCP.

Para la segunda fase, se realizó la presentación de la propuesta a los directivos, tanto de las instituciones educativas como en los centros de protección, obteniendo el aval para la implementación. De igual forma, se tramitaron las autorizaciones y los consentimientos informados con los funcionarios, docentes o empleados que accedieron a participar en el proceso.

En la tercera fase se realizaron observaciones in situ, acompañando las actividades cotidianas de los participantes,

así como entrevistas que permitieran profundizar en las categorías generadas, haciendo posible comprender los acontecimientos significativos de su vida y a afrontar los sentimientos derivados de estos.

Paralelamente se fue registrando y procesando tanto la información obtenida de las observaciones como en las entrevistas, mediante el programa Atlas $\mathrm{Ti}$, posibilitando ir definiendo categorías y posibles relaciones que fueron confrontadas con los docentes y personal de servicios, como estrategia de validación de la información.

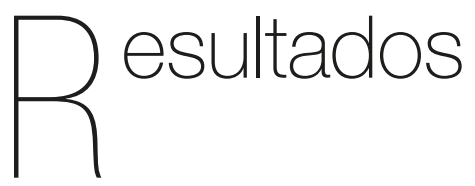

El análisis de la información obtenida arrojó tres grandes categorías que caracterizan las perspectivas sobre el cuidado de NSCP desde funcionarios, docentes y personal de servicios, en instituciones educativas y centros de protección: el cuidado como distribución de recursos, como carga laboral y como vínculo afectivo.

\section{El cuidado como distribución de recursos}


funcionarios del ICBF, podría evaluarse el cuidado como distribución de recursos, enfatizando especialmente en la mediación para asignar fondos a los programas relacionados con educación familiar y prevención, así como a las instituciones contratadas para garantizar el cumplimiento de las normas constitucionales inherentes a la protección de NSCP.

La situación descrita semeja cada vez más al rol paterno del Estado y sus agentes, que se establece en la modernidad, como proveedor de recursos, cada vez menos activo en el cuidado de sus hijos y, por tanto, cada vez más desvinculado afectivamente de ellos. Al respecto, una de las funcionarias del ICBF establece cómo el cumplimiento del rol proveedor y supervisor del recurso los aleja cada vez más de la posibilidad de trabajar cara a cara con estas poblaciones:

Nuestra carga es tanta que nos volvimos de escritorio, nosotros no podemos hacer casi acompañamientos, como todo lo están contratando [...] Nos hemos convertido en supervisores de contratos que implican mucho dinero; toca estar revisando todo, que no se le pase nada, haciendo observaciones [...] lo más triste es que nuestro trabajo, la parte pedagógica, solo cuando podemos, reunimos a los agentes educativos y les enseñamos [...] lo que realmente era valioso[...] pero lo triste es que hasta eso nos lo han limitado (cita 10:22).
El énfasis en la regulación de los procedimientos de recepción y verificación de la información y la asignación de recursos deja de lado las implicaciones emocionales del cuidado. La perspectiva del Estado enfatiza en la "organización social del cuidado" mediante procesos de estructuración del campo de las políticas y las instituciones en un conjunto interrelacionado de esferas que asumen el trabajo, la responsabilidad y el costo en la prestación de los servicios (Aguirre, 2005).

El Estado ha intentado responder a la exigencia del cuidado a NSCP asumiéndolos como un incidente irregular y como sujetos de derechos, invistiéndolos de una ideología legal densa y de procedimientos administrativos y razones asistencialistas, que en ocasiones requieren de largos períodos de espera para la asignación del cuidado familiar, bien sea en familia extensa o familia sustituta, culminando en ocasiones con la declaración de adoptabilidad. De esta forma, los NSCP pueden convertirse en objeto de conflictos ideológicos y de competitividades, ya sea desde concepciones proteccionistas o de mercado (Sánchez, 2004) que pueden propiciar la infantilización de los niños o tratarlos como si fuesen adultos (Sierra, 2004).

La Figura 1 evidencia la perspectiva del Estado que parece enfatizar en la "organización social del cuidado" mediante procesos de estructuración del campo de las políticas y las instituciones que asumen el trabajo, la responsabilidad y el costo en la prestación de los servicios (Aguirre, 2005). 


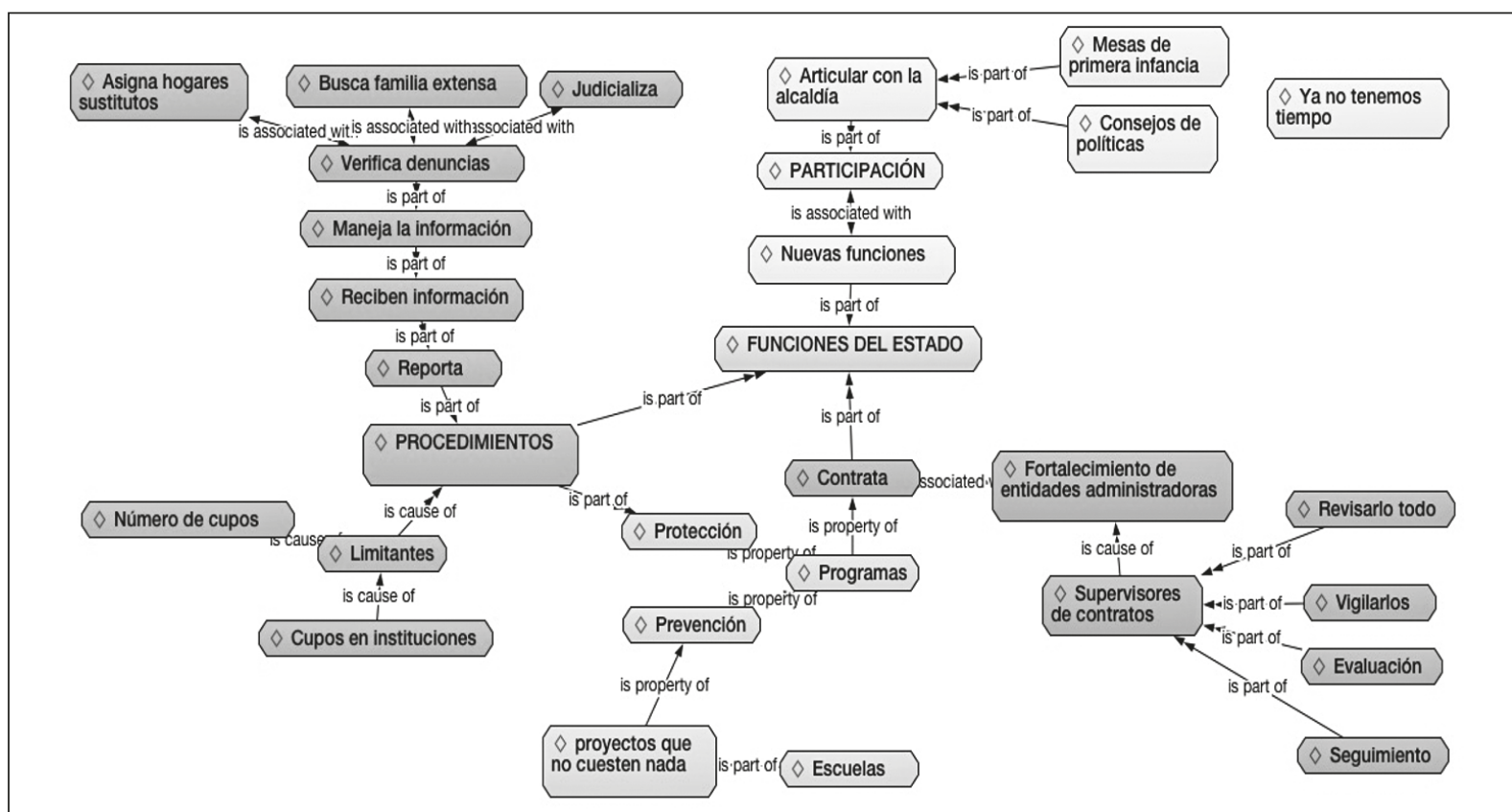

Figura 1. Entrevistas a funcionarios del ICBF y Policía Nacional.

Mirada compartida por los docentes en las instituciones educativas donde también asisten los NSCP en términos de que la prestación del servicio se convierte para el Estado en una transacción económica o un manejo político, más que en el interés por las condiciones particulares de estos niños:

El gobierno no confía en los maestros [...] yo creo que nadie administraría mejor ese dinero que el maestro, partiendo porque es que uno conoce la necesidad [...], cuando uno conoce la necesidad, uno en lugar de quitar da [...], pero entonces ellos por pagar favores políticos, entonces eso de los restaurantes escolares lo dan es los gobernadores, lo contratan con [...] usted es un amigo, usted me colocó votación, yo le voy a pagar el favor, entonces tome le doy los restaurantes escolares [...] (cita 2:56). En mi colegio, lo que está pasando es que la ración es muy pequeña para estos niños que tienen tanta hambre y que salen de la casa [...] muchos niños, el único alimento que tienen al día es el que tienen en el colegio!, porque en la casa no hay de comer [...] (cita 2:57).

Desde la perspectiva de los maestros entrevistados se evidencia cómo en el cumplimiento de las políticas relacionadas con la asignación de los recursos, en este caso para el programa de los restaurantes escolares, se presentan situaciones de corrupción en la distribución en la que los destinatarios o beneficiarios no constituyen el eje central de los programas, muy distante de las características y las necesidades sentidas por los sujetos. El reconocimiento de las situaciones particulares se hace vital para que la asignación sea realmente equitativa. En este sentido, se encuentra una gran diferencia entre el discurso y la actuación, ya que las herramientas sobre los derechos de NSCP relacionadas con las implicaciones del cuidado son controvertidas. 


\section{El cuidado como carga laboral}

El rol de cuidador que implícitamente se espera asuman los maestros en los colegios y los acompañantes en las instituciones de protección es otro de los argumentos de necesaria revisión para evidenciar las posibilidades que surgen en estos espacios donde también tienen lugar su crecimiento y donde ocurre el desarrollo emocional de los NSCP; especialmente por su directa relación con el cumplimiento de funciones laborales y las restricciones legales, así como los estereotipos y las sanciones sociales que inhiben a muchos de ellos en la posibilidad de expresar el afecto hacia ellos, aunado a las carencias formativas sobre las necesidades y formas de abordaje de los NSCP.

Los argumentos expuestos enfatizan en las dificultades que presentan los programas del Estado y los roles que se tienen que asumir como consecuencia de ellos. Según los maestros, los padres aprovechan estas circunstancias para evadir el cuidado de sus hijos que debe entonces ser cubierto de una u otra forma por ellos como una carga laboral más:

El director reunió a todos los padres de familia y les hizo una encuesta, si querían que el colegio implementara la jornada única, o sea, por la mañana y por la tarde; pues todos, a excepción de dos, porque una madre de familia era profesora y sabe cómo es el cuento y otro señor que no le convenía por el transporte, de resto, todos que sí [...]. Ya les dijo a los niños, están felices, los papitos, felices. Una señora le dijo a otra vecina, ah, yo estoy feliz, porque ahora si tengo mi tiempo a mis anchas, porque a esos chinos los mando faltando un cuarto para las siete y llegan hasta por la pura tarde [...] va, juega tejo, toma, fuma, hace de todo, porque como ya le quitaron la carga de sus hijos. [...] o sea, pues con el tiempo los papitos que van a pedir, que los guardemos ahora en la noche [...] así como los cuarteles, pues simplemente camarotes, unas duchas y que les cuidemos los niños [...] (cita 2:20).

Desde la perspectiva de los maestros, los cambios en el sistema educativo demandan nuevos roles, asignándoles tareas que no corresponden a su función de enseñanza y para las que no se tienen las competencias necesarias en el cuidado, sobre todo en NSCP que afrontan circunstancias como el maltrato o el abuso. El impacto emocional de las situaciones genera en ocasiones acciones de rechazo y denuncia cuyos efectos pueden ser adversos en el sentido de recibir amenazas o agresiones por parte de los padres o cuidadores. Finalmente, lo mejor es no enterarse, lo que implica no interesarse y alejarse afectivamente de los niños en estas condiciones:

Nosotros a veces denunciamos, por ejemplo, aquí el caso de una niña; pero entonces se gana también uno problemas [...] una profe denunció un caso porque la niña llegaba todos los días tarde, era de primaria y le vió los golpes [...]. La profe denunció y de una vez, recibe amenaza, que lambona, metida, la mamá llegó que era una atrevida, que qué le importaba, ahí si hubo tiempo, para eso sí sacan tiempo los papitos, entonces esas cosas a uno le dan miedo [...] (cita 2:26). 
De esta forma, la dimensión emocional del cuidado está permeada por el temor a tener expresiones afectivas con los estudiantes en razón a las regulaciones que se han instituido relacionadas con el acoso sexual, a pesar de reconocer que forman parte de las necesidades de cuidado en los niños. La abstención de las demostraciones de cariño como caricias o abrazos se presenta por miedo a cruzar el límite de lo permitido o a ser malinterpretado por los niños y niñas. También se señala la relevancia de alcanzar el control de las emociones, específicamente aquellas que dan un carácter negativo a las relaciones posibles en su interacción cotidiana.

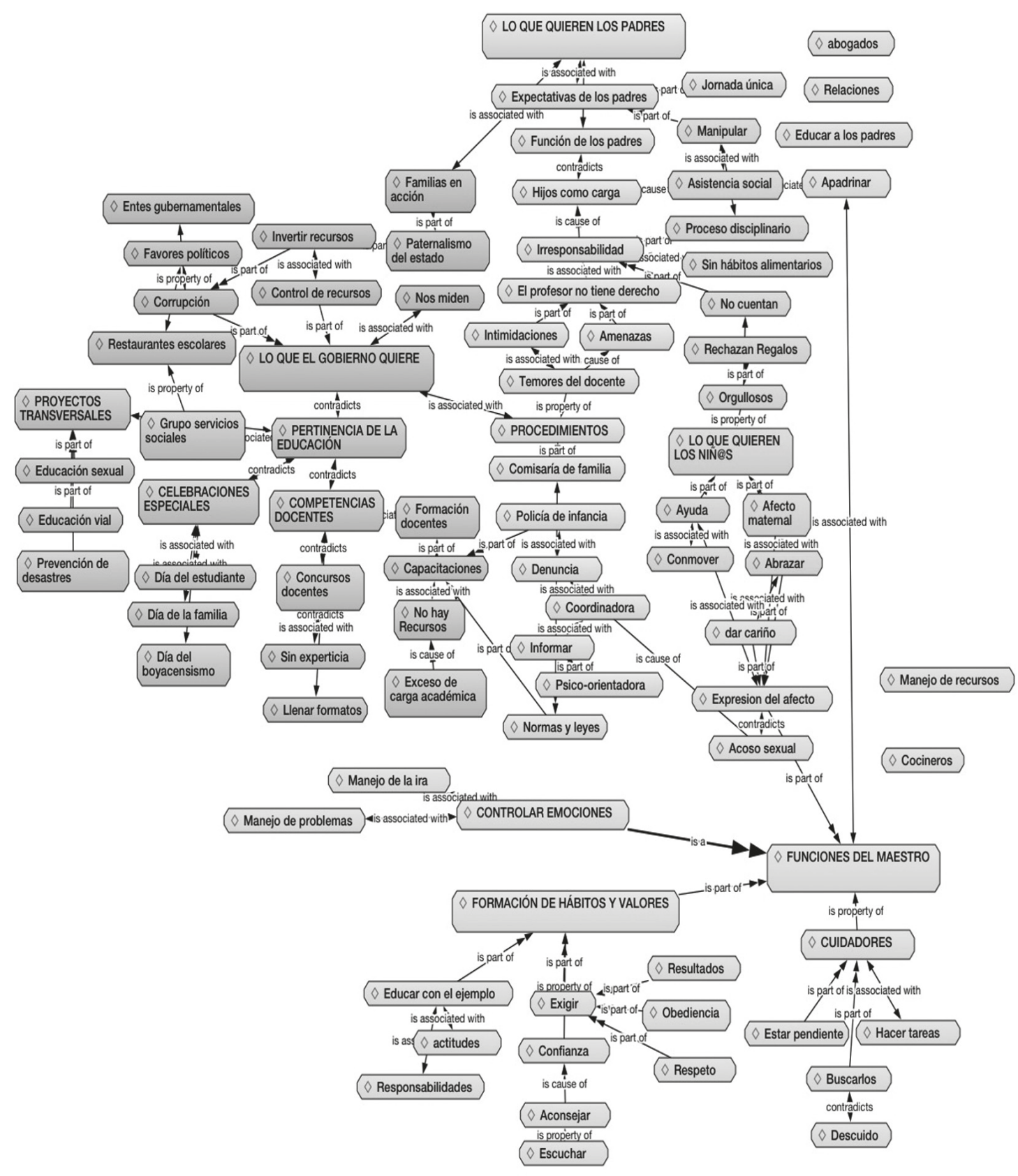

Figura 2. Entrevistas docentes del Colegio Técnico Nazareth. Fuente: Elaboración propia 


\section{El cuidado como vínculo afectivo}

Para los docentes en las instituciones de protección, pero especialmente para algunas de las personas que prestan servicios en ellas, como, por ejemplo, la cocina o jardinería, el cuidado de NSCP requiere entenderlos, en primer lugar, como sujetos con valor con una realidad propia que los confronta como humanos, se establece un vínculo afectivo en ocasiones derivado de la identificación con la propia historia. Esta forma de relación requiere de habilidades específicas asociadas a la comprensión y sensibilidad, la relación es de co-existencia y de con-vivencia:

Cuando llegó mi Camilito era muy chiquitico, daba lástima, a veces se metía ahí debajo (señala la isla o mesón), lo escondía ahí debajito o por ahí donde fuera [...] Como Camilo ha habido varios muchachos que se han apegado a mí, porque ha habido algunos que han llegado que ay Dios, algunos que incluso me han dicho mamá [...] llegaron muy pequeños, ellos se la pasaban era pa este lado, donde uno estaba, yo cocinando y ellos por ahí rondando, uno les daba algo de comida y los consentía (risas), regáleme esto, por ahí si estaba haciendo mucho frío, entonces darles algo calientico, así [...] conversaba con ellos, sobre de dónde venían, aunque como uno anda tan ocupado casi no puedo hablar con ellos, entonces solo estar ahí [...] (cita 5:22).

El cuidado involucra también una conexión personal y emocional entre los cuidadores y los NSCP, es decir, la provisión de un mínimo de vínculos sociales y afectivos intrínsecos a la condición humana, así como actividades ligadas a la educación y a la compañía (Daly \& Lewis, 2000; Folbre, 2008).

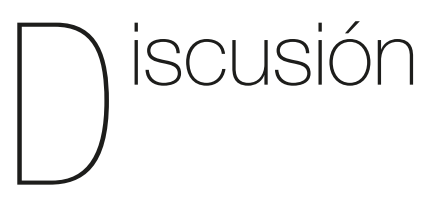

A pesar de la constante promoción de los derechos de niños, niñas y adolescentes (Pérez \& López, 2011), el abandono infantil sigue creciendo lo que plantea fuertes retos a los organismos con que cuenta la sociedad para acompañar el desarrollo de NSCP.

El cuidado que encuentran los NSCP en los ambientes educativos y de protección donde tiene lugar su crecimiento, está íntimamente ligado a las perspectivas de quienes tienen a su cargo este proceso, así como a los estados de animo que caracterizan su relación con el mundo y las comprensiones que se tienen con respecto a la condición de vulnerabilidad y dependencia como elementos constitutivos de la existencia de los NSCP (Budig \& Folbre, 2005; Pérez \& López, 2011).

Las acciones dirigidas a transformar la realidad de NSCP, más allá de la emoción particular que puedan sentir sus promotores (Blum, 1994), implica entender el cuidado como una responsabilidad social, política y moral necesaria para la sostenibilidad de la vida (Del Valle, 2003), así como la creación de condiciones de posibilidad (Pattaroni, 2003), encaminadas a reivindicar las políticas en torno a la 
infancia que contienen la responsividad como atención a la realidad efectiva de los NSCP (Terrón, 2010) e implican aspectos cognitivos y afectivos necesarios para el crecimiento humano (Noddings, 2003).

El reconocimiento de las necesidades, especialmente las de carácter afectivo de las personas (Comins, 2008), no constituyen el eje central del cuidado de los NSCP, para quienes los vínculos afectivos y relacionales que lo caracterizan, han sido atribuidos al grupo familiar desconociendo a otros actores sociales (Gilligan, 1985). Acciones ocasionales como dar una comida, ofrecer un baño o ropa, compartir alguna actividad, etc., constituyen interacciones que no generan un compromiso permanente con los NSCP, cuestionando el carácter responsivo del cuidado como una actividad de relación, de ver y responder a la necesidad, de asumir el cuidado del mundo sosteniendo la red de la conexión para que nadie se quede solo (Gilligan, 1985).

Las experiencias de cuidado ocasional constituyen una excepción a la regla general de indiferencia signada muchas veces por la sobrecarga laboral de los funcionarios de las instituciones educativas y de protección lo que indudablemente influye en la calidad del cuidado de los niños, generando así diversas formas de displicencia, desgano, apatía, lo que, a su vez, podría traducirse como desprotección. Como señala Muñoz (2010), en perspectiva responsiva, la desatención, el descuido, son, o pueden ser, de por sí, un mal moral, puesto que no responder no sería un mero vacío o cero de respuesta ni una posición neutra, sino una manera determinada de responder, que lleva consigo un esfuerzo "positivo" para no darse cuenta, para no sentirse uno concernido.

La convivencia de los sujetos dentro de un orden social coherente solo es posible en presencia de un conjunto de presupuestos legales que regulen con eficiencia los contenidos de las interacciones y los intercambios que se producen entre quienes forman parte de una sociedad determinada. Sin embargo, no basta con que las normas se hayan promulgado correctamente, es necesario reconocer la incidencia que pueden tener los intereses comunes que permiten a los individuos convivir, aceptar la existencia de otros y resolver las diferencias que pudieran existir (Latouche, 2011).

Al respecto, Noddings justifica la ética del cuidado en la propia naturaleza del ser humano que antepone las personas y sus circunstancias a los principios éticos, siguiendo un modelo más intuitivo, receptivo, no secuencial, según nuestras capacidades y propio esquema de valores: "No podemos derivar que la ética del cuidado sea una perspectiva irracional, sino que tiene su propia racionalidad" (Noddings, 1992, p. 86).

Las situaciones que enfrentan los NSCP representan condiciones particulares signadas por emociones que no se pueden descuidar durante la interacción. Ello constituye un desafío más para los organismos de control en su proceso formativo, pues exige impartir conocimientos (Fernández, 2002), que garanticen el respeto a los derechos fundamentales, las libertades públicas y seguridad ciudadana, ya que nadie quiere ser dañado por otros, ni vivir con el miedo de no ser ayudados en tiempos difíciles (Noddings, 2002). 
Como señala Vázquez (2009), la práctica del cuidado surge del reconocimiento de la fragilidad del otro, no de la obediencia a unos principios prescriptivos independientemente del rol que se cumpla en la sociedad, un deber moral más que una imposición normativa. No se trata de una ética basada en la aplicación imparcial de ciertos principios formales, sino de una respuesta afectiva y no neutral ante la necesidad y la vulnerabilidad de otros seres humanos.

Para ello, es necesario establecer los criterios con los cuales la sociedad considera el papel que cumple en la convivencia con la niñez, lo que haría posible la organización de un proyecto colectivo para su cuidado. Se trata de trascender la discusión normativa y profundizar en los aspectos que van a garantizar que las personas se acepten como parte activa y comprometida en un proyecto común, dentro del cual cada individuo es reconocido, aceptado e incorporado, en cuanto miembro valioso e importante en el crecimiento de NSCP, quienes le reclaman una serie de responsabilidades y compromisos que les son exigibles.

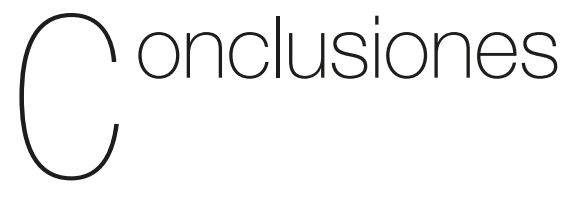

Los intercambios e interacciones espontáneas que se originan en función del cuidado de los NSCP en contextos educativos o de protección, responden al abandono o descuido de sus padres o cuidadores. Ante la ausencia de cuidado parental, la institución educativa podría constituirse en una posibilidad de la educación del carácter de los NSCP y aportar una formación que les ayude a obrar moralmente, regir su comportamiento y fortalecer su capacidad de decisión para enfrentar el mundo cotidiano. Una ética del cuidado implica para los centros educativos fomentar relaciones afectuosas, de diálogo y cuidado mutuo para lograr que los NSCP se sientan queridos y, por tanto, crezcan y lleguen a ser personas cariñosas, que aman y son amadas, lo cual es clave de cualquier educación exitosa. El sistema de educación formal es debatido en cuanto alternativa para los procesos de socialización de NSCP. El interés del Estado, centrado en la ampliación de la cobertura educativa y la ampliación de la jornada, cuestiona el conocimiento de las condiciones por las que atraviesan los NSCP, ya sea porque el incremento de estudiantes por docente sumerge cada vez más en el anonimato los casos particulares o porque los NSCP no tienen ningún interés en que su situación sea conocida por quienes conforman la comunidad educativa.

Para los centros de protección, entender las diferencias individuales y aceptar al otro como distinto es un aspecto central a tener en cuenta por parte de los cuidadores que integre aspectos intersubjetivos y factores sociales, para brindar un cuidado de calidad a los NSCP. Para ello, los cuidadores requieren de algunas capacidades fundamentales en la labor de cuidado, además de contar con recursos materiales y emocionales que les permitan orientar respuestas adecuadas para suplir las necesidades de los niños durante la infancia.

El reto consiste en establecer las formas más apropiadas de ofrecer los cuidados que necesitan y la educación que 
les permita "devenir adulto", además de construir su identidad como personas que permanecen en estrecha relación con sus entornos socioculturales. Aquí, la noción de cuidado involucra acciones y relaciones encaminadas a alcanzar los requerimientos físicos y emocionales de NSCP, así como los marcos legales y sociales que regulan su implementación.

Como fortaleza, el estudio representa una excusa para el debate necesario sobre una realidad que pervive en el mundo cotidiano de las instituciones educativas y centros de protección de cara a las políticas orientadas al cuidado de NSCP.

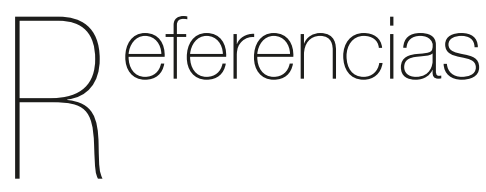

Aguirre, R. (2005). Los cuidados familiares como problema público y objeto de política. Recuperado de https://dds.cepal. org/eventos/presentaciones/2005/0628/ Rosario_Aguirre.pdf

Blum, L. (1994). Moral perception and particularity. Nueva York: Cambridge University Press.

Boff, L. (1999). Saber cuidar. México: Fondo de cultura económica.

Boff, L. (2002). El cuidado esencial. Madrid: Trotta.

Budig, M., \& Folbre, N. (2005). Activity, proximity or responsibility? Measuring parental childcare time. In N. Folbre \& M. Bittman (eds.), Family Time, The Social Organization of Care (pp 51-68). New
York: Routledge. Cambridge, MA: Harvard University Press.

Cerri, C., \& Alamillo, L. (2012). La organización de los cuidados, más allá de la dicotomía entre esfera pública y esfera privada. Gazeta de Antropología, 28(2), 1-23. Recuperado de http://hdl.handle.net/10481/23793

Comins, I. (2008). La ética del cuidado y la construcción de paz. Barcelona: Icaria Editorial.

Daly, M., \& Lewis, J. (2000). The concept of social care and the analysis of contemporary welfare states. British Journal of Sociology, 51(2), 281-298

Del Valle, T. (2003). "Contenidos y significados de nuevas formas de cuidado", Congreso Internacional Sare 2003: Cuidar cuesta: costes y beneficios del cuidado (pp. 39-61). Vitoria-Gasteiz: Emakunde/Instituto Vasco de la Mujer y Comunidad Europea Fondo Social Europeo.

Fernández, J. (2002). La reforma policial: un reto para la democracia. Santo Domingo: Tropical.

Folbre, N. (2008). Valuing Children: Rethinking the Economics of the Family, family and public policy. Cambridge, MA and London: Harvard University Press.

Gilligan, C. (1985). La moral y la teoría. Psicología del desarrollo femenino. México: FCE.

Gilligan, C. (1985). La moral y la teoría. Psicología del desarrollo femenino. México: FCE.

Heidegger, M. (2000). El ser y el tiempo (trad. José Gaos). México: FCE. 
Latouche, M. (2011). Los dilemas de Antígona. Reflexiones en torno al problema de la desobediencia civil. Episteme, 31(2), 25-44. Recuperado de http://www.scielo.org.ve/ pdf/epi/v31n2/art02.pdf

Marcus, G. (2001). Etnografía en/del Sistema Mundo. El surgimiento de la etnografía multilocal. Alteridades, 11(22), 111-127

Muñoz, J. (2010). Responsividad y cuidado del mundo. Fenomenología y ética del care. Revista Internacional de Filosofía, 49, 35-48.

Noddings, N. (1992). Variability: a pernicious hypothesis. Review of Educational Research, 62(1), 85-88.

Noddings, N. (2002). Educating moral people. A caring alternative to character education. N.Y: Teachers College Press.

Noddings, N. (2003). Happiness and education. Cambridge: Cambridge University Press.

Ortega y Gasset, J. (1986). Obras completas. Madrid: Taurus/Fundación José Ortega y Gasset, 2004-2010

Pattaroni, L. (2011). "Le care est-il institutionnalisable? Quand la "politique du care» émousse son éthique". En P. Paperman \& S. Laugier (eds.), Le Souci des autres. Éthique et politique du care (pp. 209-233). Paris : Fondation Braillard Architectes

Pérez, A., \& López, S. (2011). Desigualdades a flor de piel: cadenas globales de cuidados. Madrid: ONU Mujeres.

Pinheiro, P. (2012). Informe mundial sobre la violencia contra niños y niñas. Recuperado de http://www.unicef.org/
Red Latinoamericana de Acogimiento Familiar. [RELAF] (2011). Niñez y adolescencia institucionalizada: visibilización de graves violaciones de $D D H H$. Buenos Aires: Argentina: Publicaciones sobre niñez sin cuidados parentales en América Latina: Contextos, causas y respuestas

Robinson, F. (1999). Globalizing Care. Ethics, Feminist Theory and International Relations. Oxford: Westview Press.

Rodrígues, N., \& Franco, I. (2013). Madres e hijos imposibles: el abandono y las instituciones. Informes Psicológicos, 7, 29-38.

Sánchez, J. (2004). Orfandades infantiles y adolescentes. Introducción a una sociología de la infancia. Quito: Universidad Politécnica Salesiana, Save the Children (Suecia), Ediciones Aby-Yala.

Sierra, C. (2004). Proceso de socialización en niños abandonados e institucionalizados (Tesis de grado). Universidad de los Andes. Bogotá. Recuperado de https://biblioteca. uniandes.edu.co/visor_de_tesis/web/?Ses sionID=L1R|c2|zXzlwMDVfc2VndW5kb19z ZW1lc3RyZS8wMDAwNTQxOC5wZGY\% $3 \mathrm{D}$

Terrón, J. M. (2010). Responsividad y cuidado del mundo. Fenomenología y ética del care. Daimon. Revista Internacional de Filosofía, 49, 35-48.

Tronto, J. (2009). Un monde vulnerable. Pour une politique du care. París: Éditions la Découverte.

Tronto, J. (2010). Cura e politica democratica. Alcune premesse fondamentali. La Societá degli Individui, 38(2), 34-42. 
pp • 109-124 María Rosa Estupiñán Aponte y Fred Gustavo Manrique Abril

Unicef (2006). Estado mundial de la infancia 2006: excluidos e invisibles. Recuperado de https://www.unicef.org/spanish/ sowc06/profiles/street.php
United Nations (2010). Guidelines for the alternative care of children. New York: Unga. 